\title{
Stromal Expression of CD10 by Immunohistochemistry in Odontogenic Keratocyst (OKC), Dentigerous and Radicular Cysts and Its Correlation with Local Recurrence and Aggressive Behaviour
}

\author{
Anam Ali ${ }^{1 *}$, Muhammad Asif ${ }^{2}$, Bismah Ahmad ${ }^{2}$, Shahid Jamal ${ }^{3}$, Iram Ali ${ }^{4}$, \\ Muhammad Tahir Khadim ${ }^{1}$
}

\begin{abstract}
Objective: To assess and compare the stromal expression of CD10 in OKC, dentigerous and radicular cysts. Materials and Methods: This comparative, cross sectional study was conducted at Armed Forces Institute of Pathology (AFIP), Rawalpindi, from Jan 2017 to Dec 2017. Total sixty cases comprising 20 of each OKC, Dentigerous and Radicular cysts were included in this study. Hematoxylin and eosin (H and E) sections were performed followed by immunohistochemical staining for CD10 antibody. Expression of CD10 was evaluated and compared. Results were analyzed by using SPSS version 20.0. Chi Square test was performed with $\mathrm{P}$ value $<0.05$ was considered as significant. Results: A total of 60 cases, 20 of each OKC, dentigerous and radicular cysts were taken. In our study, $38(63.3 \%)$ male and $22(36.7 \%)$ female patients with the mean age of $32 \pm 15$ (mean \pm SD) were included. Percentage of CD10 positive cells were highest in sub-epithelial stroma of OKC (95\% cases) as compared to radicular and dentigerous cysts (60 and $70 \%)$ with highest number of cases showing intense staining in OKC 13(65\%) as compared to other odontogenic cysts i-e 4(20\%) and $2(10 \%)$ respectively. There was a statistically significant association between odontogenic cysts and proportional score, intensity score and combined score of stromal $\mathrm{CD} 10$ expression $(\mathrm{P}=0.009, \mathrm{p}=0.001$ and $\mathrm{p}=0.000)$. Conclusion: In this study, we found that highest stromal CD10 expression in $\mathrm{OKC}$ as compared to dentigerous and radicular cyst, which might be due to aggressive behaviour and increased risk of recurrence in OKC. Expression of CD10 marker will further aid the clinician to plan appropriate surgical intervention and keep regular follow-ups to identify recurrences.
\end{abstract}

Keywords: CD10- Odontogenic Keratocyst (OKC)- dentigerous cysts- radicular cyst

Asian Pac J Cancer Prev, 20 (1), 249-253

\section{Introduction}

Odontogenic cysts (OCs) are the commonest forms of cystic lesions that involve the maxillofacial area (Bhakhar et al., 2016). These are fluid filled pathological spaces, which are lined by odontogenic epithelium. They are further categorized as developmental and inflammatory cysts (Naz et al., 2012). Radicular cysts labelled as inflammatory odontogenic cysts comprises 52 to $68 \%$ of entire cystic lesion affecting the jaw (Nainani et al., 2014). Dentigerous cysts are most frequently associated with impacted third molar and accounts for $20 \%$ of all cystic jaw lesions (Paul et al., 2013). This complicated group of lesions have different presentation. Some may be detected accidently because of small innocuous lesion but others may be very destructive and may even show malignant transformation. Among destructive types, most common are odontogenic keratocyst (Nayak et al., 2013). In 2005 WHO classification of head and neck tumor, OKC was reclassified and changed to keratocystic odontogenic tumor (KOT) because of its high recurrence rate, aggressive nature and specific histological features. But a short time ago, keratocystic odontogenic tumor was recategorized again into cystic lesions by WHO 2017 classification of head and neck tumors. Therefore, it is a matter of discussion since many decades about the nature of $\mathrm{OKC}$ as either tumor or cyst. But still OKC deserve special attention due to its high recurrence rates (25-60\%), locally aggressive behaviour, destructive nature, evidence of chromosomal and genetic abnormalities and high mitotic activity are often found in this lesion as seen in neoplasia as compared to other cysts such as dentigerous 
and radicular cysts (Deepak et al., 2017).

CD10 is a zinc dependent, cell surface metalloendopeptidase, which can be used as a progression marker. Basically, it acts as a double edge sword. Its physiological function is to deactivate and degrade a wide variety of biologically active peptides. However, in most of malignancies, expression of CD10 in stroma is associated with high grade tumor and increased risk of recurrence (Hormozi et al., 2016; Mishra et al., 2016). So, the objective of this study to investigate and compare the stromal expression of CD10 in OKC, dentigerous and radicular cyst.

\section{Materials and Methods}

This comparative, cross sectional study was carried out in Histopathology department, Armed Forces Institute of Pathology Rawalpindi from Jan 2017 to Dec 2017. Approval of institutional review board was taken. Non-Probability convenience sampling was done. A total of 60 cases were retrieved from the record files along with their paraffin embedded blocks, comprising 20 each of OKC, Dentigerous and Radicular cyst of both genders of all age groups. Scanty, poorly fixed specimen and all benign cysts other than odontogenic cysts were excluded from study.

The data regarding gender, age, site and laterality of the cyst were recorded from the clinical histories of each case. Paraffin embedded blocks were trimmed and cut into thin sections of 3-5 microns using microtome and mounted on slides. Hematoxylin and eosin (H\&E) staining was performed on tissue section slides. Histopathological diagnosis of each case was made using under the light microscope.

For immunohistochemical technique, ready to use kit of Monoclonal antibody CD10 (56C6, IgG1) was obtained from Leica Biosystem laboratories (UK). IHC staining of CD10 was performed on each case as per manufacturer guideline. Results were analyzed by using SPSS version 20 .

\section{Evaluation of CD10 Staining}

Evaluation of stromal CD10 expression was scored at 10 high power field (x 400) as described by Aziz and Amin, (2012).16 CD10 positive stromal cells were defined as brown membranous and cytoplasmic staining. Membranous staining of lymphocytes in germinal center of lymph node was taken as positive control. Proportion, Intensity and combined score were evaluated as per following criteria.

Proportion Score $(P)$ :

$$
\begin{gathered}
0 \text {--- }<0 \% \\
1 \text {--- } 10-25 \% \\
2---25-50 \% \\
3 \text {--- }>50 \% \\
\text { Intensity score (I): } \\
0 \text {--- Negative } \\
\text { 1---Weak staining } \\
\text { 2---Moderate staining } \\
\text { 3---Intense staining }
\end{gathered}
$$

Combined Score (T)

Combined score $(\mathrm{T})=$ Proportion score $(\mathrm{P})+$ Intensity score (I)

Score $0-1=$ Negative

Score $2=1+$

Score $3-4=2+$

Score $5-6=3+$

\section{Results}

A total of 60 cases comprising 20 of each $\mathrm{OKC}$, dentigerous and radicular cyst were studied. In our study, $38(63.3 \%)$ male and $22(36.7 \%)$ female patients with the mean age of $32 \pm 15$ (mean \pm SD) were studied. Mandible was the most commonly affected site in 37 (61.7\%) cases followed by maxilla, which was involved in 23 (38.3\%) cases. Among 60 cases of odontogenic cysts, the proportion and intensity scores of CD10 was studied

\begin{tabular}{|c|c|c|c|c|c|}
\hline Stromal Immunoreactivity of CD10 & $\begin{array}{c}\text { OKC } \\
\mathrm{n}=20 \text { (Percentage })\end{array}$ & $\begin{array}{c}\text { DCs } \\
n=20 \text { (Percentage })\end{array}$ & $\begin{array}{c}\text { RCs } \\
n=20 \text { (Percentage })\end{array}$ & p-value & Results \\
\hline \multicolumn{6}{|l|}{ Percentage of CD10 positive cells (p) } \\
\hline$<10 \%$ & $1(5)$ & $5(25)$ & $8(40)$ & & \\
\hline $10-25 \%$ & $4(20)$ & $10(50)$ & $8(40)$ & & \\
\hline $25-50 \%$ & $12(60)$ & $4(20)$ & $3(15)$ & & \\
\hline$>50 \%$ & $3(15)$ & $1(5)$ & $1(5)$ & 0.009 & Significant \\
\hline \multicolumn{6}{|l|}{ Intensity of CD10 positive cells (I) } \\
\hline Negative & $1(5)$ & $5(25)$ & $8(40)$ & & \\
\hline Weak & $4(20)$ & $7(35)$ & $10(50)$ & & Significant \\
\hline Moderate & $2(10)$ & $4(20)$ & $0(0.0)$ & & \\
\hline Intense & $13(65)$ & $4(20)$ & $2(10)$ & 0.001 & \\
\hline
\end{tabular}
(Table-1). Out of 20 cases of OKCs, combined score of $3+$ was obtained in maximum number of 11 (55\%) cases, while $8(40 \%)$ cases were scored as $2+$ and only $1(5 \%)$ case was negative. On the other hand, Dentigerous cysts showed negative score in $5(25 \%)$ cases, $1+$ score in 7 $(35 \%)$ of cases, $2+$ score was obtained in $5(25 \%)$ cases and only $3(15 \%)$ cases showed of $3+$. While in radicular

Table 1. Immunoreactivity of CD10 (Number+ Intensity) Positive Cells in Sub Epithelial Stroma of OCs

OCs, Odontogenic cysts; OKC, Odontogenic keratocyst; DCs, Dentigerous cysts; RCs, Radicular cysts. 

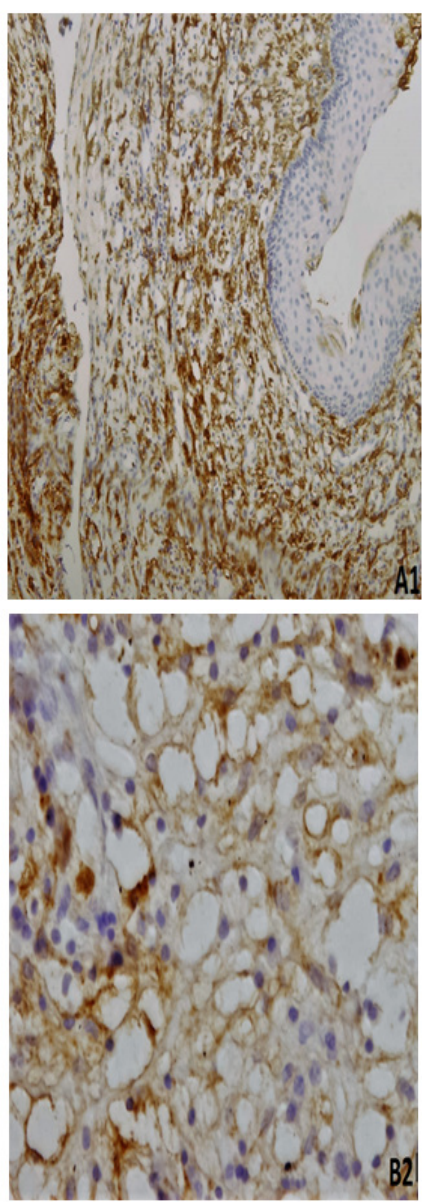
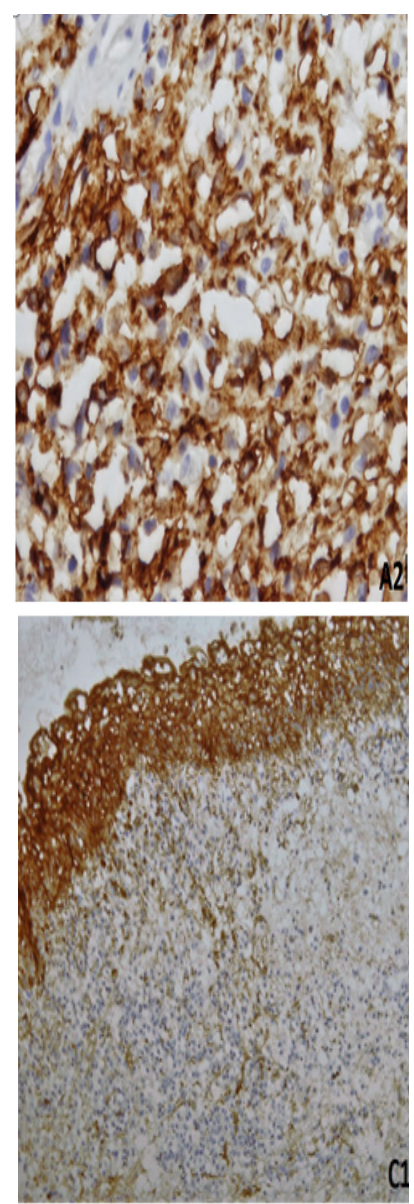
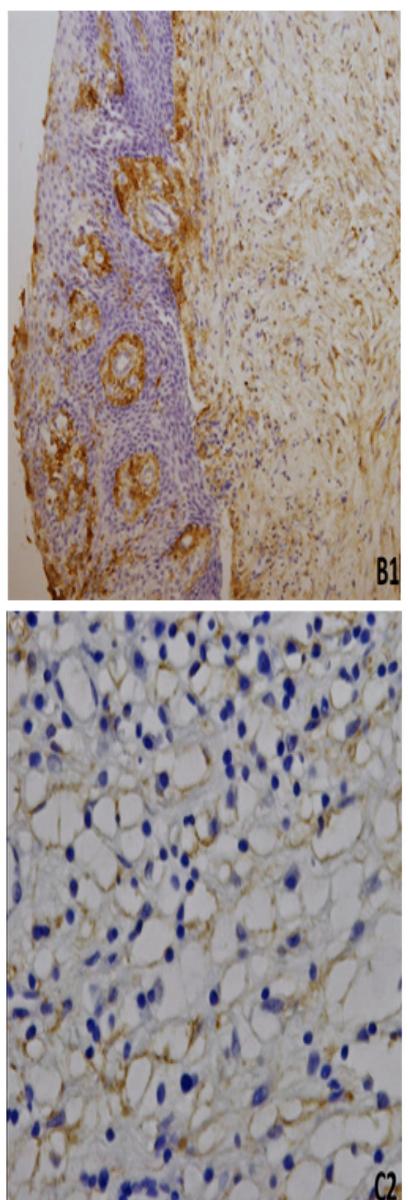

Figure A1,A2. CD10 immunostaining in the stroma of $\mathrm{OKC}$ : This image represents cytoplasmic and membranous pattern of strong intensity staining in about $>50 \%$ cells $(\mathrm{A} 1=100 \mathrm{x}$ magnification, $\mathrm{A} 2=400 \mathrm{x}$ magnification).

Figure B1, B2. CD10 immunostaining in the stroma of Dentigerous cyst: This image represents cytoplasmic and membranous patterns of moderate intensity staining in about $10-25 \%$ cells $(B 1=100 x$ magnification, $B 2=400 x$ magnification).

Figure C1, C2. CD10 immunostaining in the stroma of Radicular cyst: This image represents cytoplasmic and membronous pattern of weak intensity in about $<10 \%$ cells $(C 1=100 x$ magnification, $C 2=400 x$ magnification $)$.

cyst, $8(40 \%)$ of cases were negative, $9(45 \%)$ scored as $1+$, only $1(5 \%)$ and $2(10 \%)$ cases scored as $2+$ and $3+$ respectively. There is a statistically significant association between combined score of CD10 in OKC, Dentigerous and Radicular cyst $(\mathrm{P}=0.000)$.

\section{Discussion}

In oral and maxillofacial pathology, odontogenic lesions have essential clinicopathological aspects (Tekkesin et al., 2016). These are comparatively common and in any pathology service, they account for a significant number of total received biopsies (Nayak et al., 2013).
In the past, many IHC markers were used to analyse the aggressive behaviour of these cysts. The CD10 is one of them, which can be used to evaluate the aggressive nature of these lesions (Deepa et al., 2014).

In our study, percentage of stromal CD10 positive cells was greater in $\mathrm{OKC}$ as compared to radicular and dentigerous cyst. These results are comparable with the studies done by Deepa et al., (2014), and Tadbir et al., (2013), who reported increased number of CD10 positive cells in subepithelial stroma of OKC as compared to other odontogenic cysts.

In the present study, intensity of staining was also strong in majority of cases of OKCs i.e $65 \%$ than other

Table 2. Comparison of CD10 Expression in the Stroma of OCs in Different Demographic Areas

\begin{tabular}{|c|c|c|c|c|c|c|c|c|c|}
\hline \multirow[t]{2}{*}{ S.\# } & \multirow[t]{2}{*}{ Studies } & \multirow[t]{2}{*}{$\begin{array}{c}\text { Demographic } \\
\text { Areas }\end{array}$} & \multirow[t]{2}{*}{$\begin{array}{l}\text { Sample } \\
\text { Size }\end{array}$} & \multicolumn{3}{|c|}{$\begin{array}{c}\text { No. of cases showing }>10 \% \text { CD } 10 \\
\text { positive cells. } n \text { (percentage) }\end{array}$} & \multicolumn{3}{|c|}{$\begin{array}{l}\text { No. of cases with strong intensity } \\
\text { of staining. } n \text { (percentage) }\end{array}$} \\
\hline & & & & OKCs & DCs & RCs & OKCs & DCs & $\mathrm{RCs}$ \\
\hline 1 & Deepa et al., (2014) & India & 60 & $14(70)$ & $2(10)$ & $2(10)$ & $12(60)$ & $6(30)$ & $8(40)$ \\
\hline 2 & Karimi et al., (2016) & Iran & 22 & - & - & - & $2(18.8)$ & - & $0(0.0)$ \\
\hline 3 & Tadbir et al., (2013) & Malaysia & 40 & $16(80)$ & $1(5)$ & - & Not mentioned & & \\
\hline 4 & Ali et al., (2018) & Pakistan & 60 & $19(95)$ & $15(75)$ & $12(60)$ & $113(65)$ & $4(20)$ & $2(10)$ \\
\hline
\end{tabular}

OCs, Odontogenic cysts; DCs, Dentigerous cysts; RCs, Radicular cysts 
cysts. This increased expression of CD10 in the stroma from radicular cyst to dentigerous cyst to OKC might explain the different behaviour of these lesions and locally aggressive nature of OKC. Deepa et al., (2014) and karimi et al., (2016) noted strong intensity of stromal CD10 expression in $\mathrm{OKC}$ as compared to other odontogenic cysts. These finding are consistent with the results of our study.

Hormozi et al., (2013) in their recent study proposed that absence of any significant difference of CD10 expression in stroma of ameloblastoma and OKC might be due to neoplastic potential and high recurrence rate. Stroma of most host cells contain tumor-suppressing abilities but during malignant process, stroma will change and eventually support invasion, metastasis and growth (Bremnes et al., 2011). Singh et al., (2015) showed that amount of glycosaminoglycans and density of collagen fibres in different zones were similar in radicular and dentigerous cysts. Although in case of $\mathrm{OKC}$, unattached collagen fibres and increased amount of glycosaminoglycan contents were present as compared to dentigerous and radicular cyst. They also suggested that $\mathrm{OKC}$ possesses different ground substances and collagenous pattern, which is most likely due to the aggressiveness of $\mathrm{OKC}$.

Aziz and Amin (2014) conducted a study, reported that the expression of ki-67 and CD10 together with histological assessment can be used as a helpful marker to know the biological behaviour of tumors and also mentioned that expression of CD10 and ki-67 were significantly associated with high recurrence rate in odontogenic lesions ( $\mathrm{P}$ value $=0.000,0.003$ respectively). They also reported that the solid ameloblastoma showed high CD10 positivity than the peripheral and unicystic variants. Helmy and his co-workers observed that in oral squamous cell carcinoma, stromal expression of CD10 increased from well to poorly differentiated form $(p<0.05)$ and can be a predictive of worse prognosis (Helmy et al., 2015). Another recent study by Bassyoni et al., (2017) identified that CD10 expression can also be used for the assessment of growth rate, metastasis and invasion of gastric carcinoma, which was found to be increased as the disease advances. Ulaganathan et al., (2017) found that high expression of CD10 in subepithelial stroma of breast cancer is directly proportional to tumor grade and proposed that CD10 can be used as a new prognostic marker and for the advancement of new drugs. These studies proposed that the tumors with higher grades exhibited high CD10 expression in subepithelial stroma. Mishra et al., (2016) suggested that CD10 can be used as very useful progression marker. Analysis of its routine expression in addition to other markers might be very useful in the identification of aggressiveness of lesion and treatment response. Expression of CD10 showed significant correlation with high proliferative index, increased tumor size, and metastasis.

In Pakistan, it was first study to be conducted in AFIP on the stroma of OKC, Dentigerous and Radicular cysts. However, many studies have been conducted on epithelial lining of odontogenic cysts. Among Odontogenic cysts, $\mathrm{OKC}$ has relatively high potential for malignant transformation. We believe that further studies to explore frequency of malignant transformation in these odontogenic lesions and its association with stromal CD10 should be carried out in larger series to find the premalignant potential of these notorious lesions.

In conclusion, we found that highest stromal CD10 expression in OKC as compared to dentigerous and radicular cyst, which might be due to aggressive behaviour and increased risk of recurrence in OKC. Expression of CD10 marker will further aid the clinician to plan appropriate surgical intervention and keep regular follow-ups to identify recurrences.

\section{References}

Abdel-Aziz A, Amin MM (2012). EGFR, CD10 and proliferation marker Ki67 expression in ameloblastoma: possible role in local recurrence. Diag Pathol, 7, 14.

Bassyoni OY, Nasif SN (2017). Tissue expression of CD10 and $\mathrm{CD} 15$ proteins in gastric lesions: correlation with clinicopathological features. EJP, 37, 321-7.

Bhakhar VP, Shah VS, Ghanchi MJ, et al (2016). A comparative analysis of cytokeratin 18 and 19 expressions in odontogenic keratocyst, dentigerous cyst and radicular cyst with a review of literature. JCDR, 10, 85.

Bremnes RM, Dønnem T, Al-Saad S, et al (2011). The role of tumor stroma in cancer progression and prognosis: emphasis on carcinoma-associated fibroblasts and non-small cell lung cancer. JTO, 6, 209-17.

Deepa K, Munisekhar M, Suri C, et al (2014). Comparision of immunohistochemical expression of CD10 in odontogenic cysts. JCDR, 8, ZC35.

Deepak Passi DS, Mahinder S, et al (2017). Odontogenic keratocyst (OKC) or keratocystic odontogenic tumor (KCOT) Journey of OKC from cyst to tumor to cysts again: Comprehensive Review with recent updates on WHO classification (2017). IJCR, 9, 6.

GS TB, Ulaganathan S, Venkatachalam AS, et al (2017). An analysis of stromal expression of CD10 in invasive ductal carcinoma of breast and its correlation with histological grade. IJRMS, 5, 1629-35.

Helmy IM, Baghdady HM, Omar S (2015). Immunohistochemical Expression of Cd10 in Oral Squamous Cell Carcinoma. $J$ Dent $S c i, 425,1-7$.

Hormozi E, Fard VN, Naseri MA, Jahromi NH, Keshani F (2016). Comparison of immunohistochemical expression of CD10 in keratocystic odontogenic tumor and ameloblastoma. Dent Res J, 13, 110.

Karimi B, HB MS (2016). Immunohistochemical comparison of $\mathrm{CD} 10$ expression in ameloblastoma, odontogenic keratocyct and radicular cyst. IJCRCPS, $\mathbf{3}, 7$.

Mishra D, Singh S, Narayan G (2016). Role of B cell development marker CD10 in cancer progression and prognosis. Mol Biol Int, 2016, Article ID 4328697, 9 pages. http://dx.doi.org/10.1155/2016/4328697.

Nainani P, Sidhu GK (2014). Radicular cyst-an update with emphasis on Pathogenesis. JMDS, 2, 97-101.

Nayak MT, Singh A, Singhvi A, Sharma R (2013). Odontogenic keratocyst: what is in the name?. J Nat Sc Biol Med, 4, 282.

Naz II, Akhtar MKMF, Gillan SM (2012). Clinicopathological analysis of Odontogenic Cysts in a selected Pakistani population. Biomedica, 28, 61-5.

Paul R, Paul G, Prasad RK, et al (2013). Appearance can be deceptive: Dentigerous cyst crossing the midline. Nati $J$ Maxillofac Surg, 4, 100.

Singh P, Rakheja M, Agrawal N, et al (2015). The capsular 
connective tissue stroma plays a vital role in the behaviour of odontogenic. Int J Dent Med Res, 1, 31.

Tadbir AA, Geramizadeh B, Ranjbaran H (2013). CD10 expression in dentigerous cyst, odontogenic keratocyst and ameloblastoma. Asian J Biol Sci, 6, 221-7.

Tekkesin MS, Tuna EB, Olgac V, Aksakallı N, Alatlı C (2016). Odontogenic lesions in a pediatric population: Review of the literature and presentation of 745 cases. Int J Pediatr Otorhinolaryngol, 86, 196-9.

\section{(c) (i) 8}

This work is licensed under a Creative Commons AttributionNon Commercial 4.0 International License. 